\title{
A review in radiomics: Making personalized medicine a reality via routine imaging
}

\author{
Julien Guiot $^{1}$ | Akshayaa Vaidyanathan ${ }^{2,3}$ | Louis Deprez ${ }^{4}$ | \\ Fadila Zerka $^{2,3}$ | Denis Danthine ${ }^{4}$ | Anne-Noelle Frix ${ }^{1}$ | \\ Philippe Lambin $^{3}$ | Fabio Bottari ${ }^{2}$ (1) | Nathan Tsoutzidis ${ }^{2}$ | \\ Benjamin Miraglio $^{2}$ | Sean Walsh ${ }^{2}$ | Wim Vos ${ }^{2}$ | \\ Roland Hustinx $^{5,6}$ | Marta Ferreira ${ }^{6}$ | Pierre Lovinfosse ${ }^{5}$ \\ Ralph T.H. Leijenaar ${ }^{2}$ (D)
}

${ }^{1}$ Department of Pneumology, University Hospital of Liège, Liège, Belgium

${ }^{2}$ Radiomics (Oncoradiomics SA), Liège, Belgium

${ }^{3}$ The D-Lab, Department of Precision Medicine, Department of Nuclear Medicine, GROW-School for Oncology, Maastricht University, Maastricht, The Netherlands

${ }^{4}$ Department of Radiology, University Hospital of Liège, Liège, Belgium

${ }^{5}$ Department of Nuclear Medicine and Oncological Imaging, University Hospital of Liege, Liege, Belgium

${ }^{6}$ GIGA-CRC in vivo imaging, University of Liège, Liège, Belgium

\section{Correspondence}

Ralph T.H. Leijenaar, Radiomics (Oncoradiomics SA), Liège, Belgium.

Email: ralph.leijenaar@radiomics.bio

\section{Funding information}

TRANSCAN Joint Transnational Call 2016, Grant/Award Numbers: JTC2016 CLEARLY $\mathrm{n}^{\circ}$, UM 2017-8295; SME Phase 2, Grant/Award Number: RAIL n673780; ERC advanced, Grant/Award Numbers: ERC2018-PoC: 813200-CL-IO, ERC-2020-PoC: 957565-AUTO.DISTINCT, ERC-ADG-2015 nº

\begin{abstract}
Radiomics is the quantitative analysis of standard-of-care medical imaging; the information obtained can be applied within clinical decision support systems to create diagnostic, prognostic, and/or predictive models. Radiomics analysis can be performed by extracting hand-crafted radiomics features or via deep learning algorithms. Radiomics has evolved tremendously in the last decade, becoming a bridge between imaging and precision medicine. Radiomics exploits sophisticated image analysis tools coupled with statistical elaboration to extract the wealth of information hidden inside medical images, such as computed tomography (CT), magnetic resonance (MR), and/or Positron emission tomography (PET) scans, routinely performed in the everyday clinical practice. Many efforts have been devoted in recent years to the standardization and validation of radiomics approaches, to demonstrate their usefulness and robustness beyond any reasonable doubts. However, the booming of publications and commercial applications of radiomics approaches warrant caution and
\end{abstract}


694812 - Hypoximmuno; H2020 European Research Council, Grant/Award Numbers: CHAIMELEON $n^{\circ}$ 952172, EuCanlmage $n^{\circ}$ 952103, ImmunoSABR $n^{\circ}$ 733008, MSCAITN-PREDICT $\mathrm{n}^{\circ}$ 766276; Interreg V-A Euregio Meuse-Rhine, Grant/Award Number: EURADIOMICS n EMR4 proper understanding of all the factors involved to avoid "scientific pollution" and overly enthusiastic claims by researchers and clinicians alike. For these reasons the present review aims to be a guidebook of sorts, describing the process of radiomics, its pitfalls, challenges, and opportunities, along with its ability to improve clinical decisionmaking, from oncology and respiratory medicine to pharmacological and genotyping studies.

\section{KEYWORDS}

artificial intelligence, deep learning, machine learning, personalized medicine, radiomics

\section{1 | INTRODUCTION}

Imaging is a fundamental technology in medicine and is used in clinical practice to aid decision-making for screening, diagnostic, ${ }^{1}$ therapeutic, ${ }^{2}$ and follow-up purposes. Radiomics was born in 2012 as an innovative approach to image analysis, using automated high-throughput extraction of large amounts of quantitative features from standard-of-care medical images. ${ }^{3,4}$ The hypothesis is that quantitative analysis of medical image data can provide complementary information to aid physicians in the decision-making process, aided by automatic or semiautomatic software, in a fast and reproducible way. ${ }^{5}$ Radiomics is the result of several decades of computer-aided diagnosis, prognosis, and therapeutics research. ${ }^{6,7}$ A robust radiomics approach consists in the identification of a wide variety of quantitative features from medical images, the storage of such data in several independent databases functioning as a single entity (federated databases) ${ }^{8}$ and the subsequent data mining to obtain clinically relevant outcomes. ${ }^{9}$ Medical images such as CT, MR, and/or PET scans can be analyzed and processed to extract relevant radiomics features which can be used for screening, diagnostic, ${ }^{10}$ follow-up, and prognostic ${ }^{11}$ purposes as well as for pharmacokinetic and pharmacodynamic studies. ${ }^{12-14}$ Databases that collect and cross-reference vast amounts of radiomics data along with other relevant patient information from millions of cases are already a reality but still present considerable management problems. ${ }^{15-18}$ However, Radiomics is not the "philosopher stone" for clinical decision-making. Since its inception in 2012, the number of radiomics publications has grown exponentially (Figure 1) as well as its detractors and disbelievers. The proven efficacy of radiomics approaches and the enthusiasm around this new method have to be tempered by its informed application and the careful evaluation of its real potential.

Two main approaches are used for radiomics analysis, hand-crafted features and deep learning (DL). Radiomic hand-crafted features (such as intensity, shape, texture or wavelet) offer information on the specific area of the imaging scan one wishes to investigate, might be a tumor region or a whole organ. These features are distinct yet interconnected to other data sources (such as clinical, treatment or genomic data). ${ }^{19}$ The main challenge lies in the collection and integration of multimodal data sources in a quantitative fashion, delivering unambiguous clinical information and in turn allowing accurate and robust outcome prediction. ${ }^{20}$ Deep learning methods instead use a data-driven approach for model creation, mimicking simplified brain neuron interactions. Deep learning has the advantage of not needing prior segmentation of the imaging scan: however the "black box" approach of DL, that is, the lack of interpretability of the models and features generated is seen as the main limitation for clinical applicability. Moreover, DL approaches need a large amount of data to truly express their potential, and sometimes the patients' cohort available, for example in the case of rare diseases, are not enough to leverage a DL architecture in an effective manner. 


\section{Documents by year}

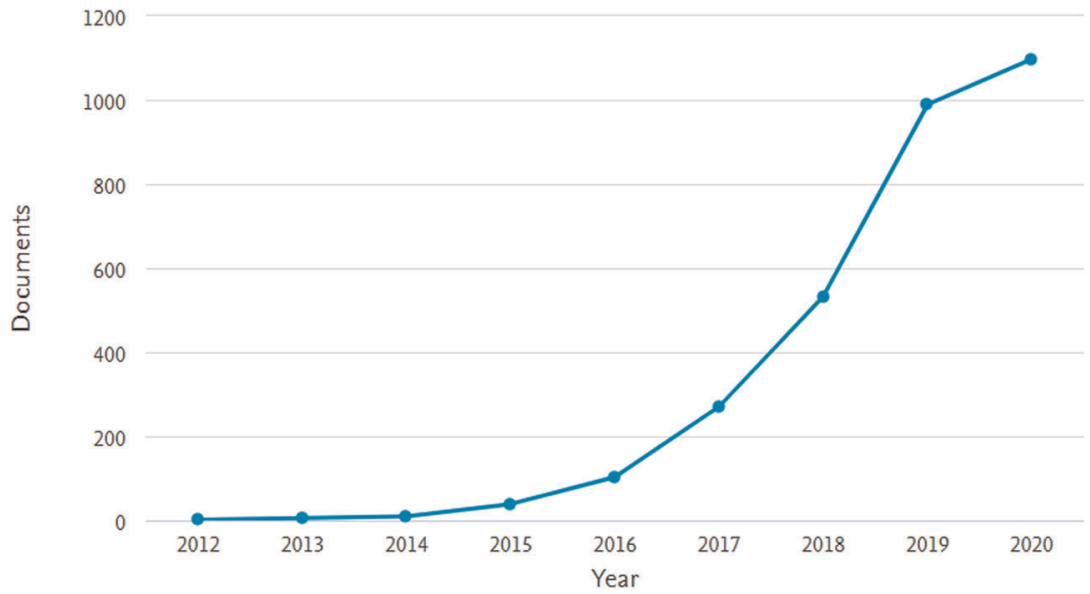

FIGURE 1 Number of "radiomics" publications per year (2012-2020). Data obtained from Scopus (09/09/ 2020) [Color figure can be viewed at wileyonlinelibrary.com]

For as much as this scenario seems straightforward and most alluring for clinicians, there are still too many published prediction models which lack standardized evaluation of their performance, reproducibility, and/or clinical utility. ${ }^{21,22}$

In this review, the pitfalls and challenges along with the opportunities presented by radiomics to improve personalized precision medicine will be showcased, stressing important methodological aspects of radiomics prediction model both in terms of development and validation. We will explore the advanced information technologies that are essential for the simultaneous management of radiomics and clinical data. Finally, we will present our outlook on the necessary steps that still need to be taken to ensure widespread acceptance of radiomics in current clinical practice.

\section{2 | GOOD PRACTICES IN RADIOMICS STUDIES}

Radiomics can be defined as a collection of methods (algorithms) used to extract a large number of features from radiographic medical images. ${ }^{9}$ Radiomics emerged originally in the field of oncology ${ }^{1,23}$; however, it can be applied to any medical study where a disease or a condition can be imaged. ${ }^{24-27} \mathrm{~A}$ radiomics study can be divided into four main phases: data selection and curation, features extraction, exploratory analysis, and modeling. Below we report a typical step-by-step radiomics workflow (Figure 2).

\section{1 | Data selection and curation}

Radiomic analysis starting point is the selection of an imaging technique (CT, MRI, PET, etc.), the identification of the region or volume of interest (ROI or VOI), and the choice of a specific prediction target-the relevant clinical question that the radiomics analysis aims to answer. In a typical oncological study, the whole primary tumor is analyzed and linked to available data on treatment outcomes and disease prognoses, such as survival rate or tumor shrinkage. Radiomic analyses can be performed on subregions of the tumor (habitats), metastatic lesions, as well as in normal tissues. Radiomics analysis, however, is not restricted to radiotherapy and can be applied to any image 


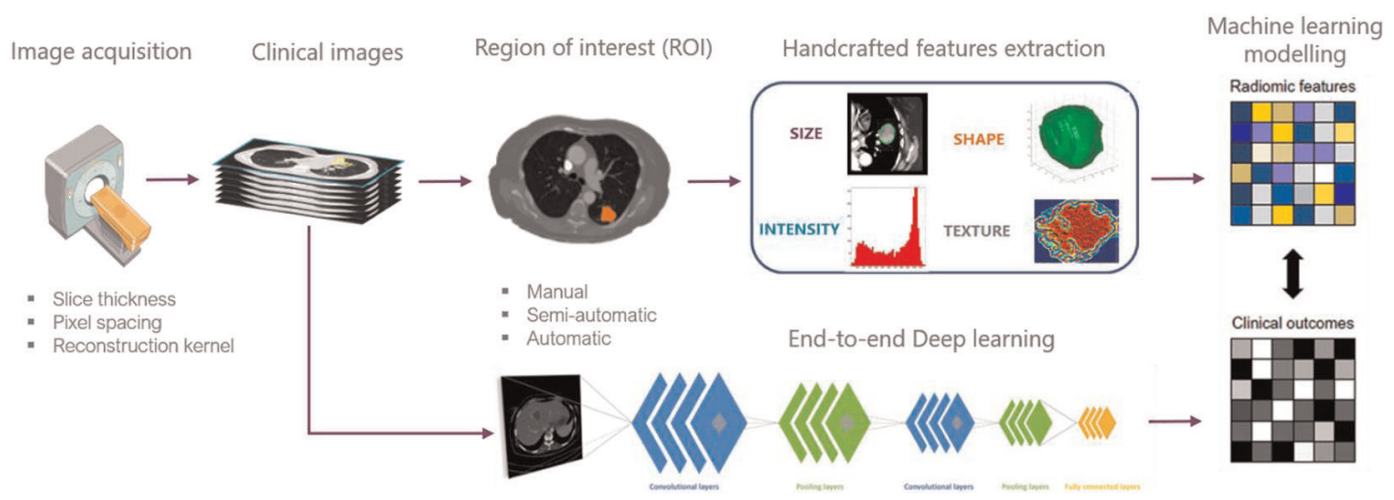

FIGURE 2 Scheme of the radiomics workflow for hand-crafted features (top) and deep learning (bottom) [Color figure can be viewed at wileyonlinelibrary.com]

generated in the clinical setting. ${ }^{28-30}$ The use of standardized imaging protocols to eliminate unnecessary variability is of paramount importance ${ }^{9,31}$ and has been recognized through the years as one of the main factors leading to low-quality radiomics analysis. ${ }^{32}$ Still nowadays, however, non-standardized imaging protocols are commonplace: reproducibility and comparability of radiomic studies would immensely benefit from clear guidelines on how imaging protocols should be applied and reported. To at least partially overcome these issues, images datasets must be carefully evaluated and where possible standardized, following well-established radiomics criteria. $^{33}$ Selection of slice increment and reconstruction kernel can be used, for example, as criteria to include or exclude imaging scans for radiomics feature extraction.

\subsection{1 | Medical imaging}

\section{Segmentation}

Segmentation is the first fundamental step in radiomics analysis and can be performed manually by expert radiologists/clinicians or (semi-) automatically. ${ }^{34}$ Both approaches have their pros and cons and the most suited one varies on a case-by-case basis. ${ }^{35,36}$ In general, automatic segmentation is more reproducible and faster than handmade segmentation. The segmentation step determines which voxels within an image are analyzed: it is easy to see that the variability in segmentation (both human- and machine-driven) can introduce bias in the evaluation of the derived radiomic features. ${ }^{37}$ For example, a semi-automatic segmentation method can result in different radiomic features than a manual segmentation, as well as segmentation performed by two different physicians. Comparing multiple segmentation approaches might be a solution to limit the amount of this bias. ${ }^{38}$ It may consist in different clinical experts manual segmentation or the perturbation of the automatic or semiautomatic segmentations with noise ${ }^{39}$ or again the combination of different segmentation algorithms. ${ }^{40,41}$

\section{Phantom studies and feature stability}

Another source of variability in the preliminary radiomics phase is the inter-machine and inter-vendor differences between the scanners employed. ${ }^{42}$ In most real-life situations, the radiomics study must rely on data acquired on different scanners from different producers and with different "history": thus, not taking into account this systematic source of uncertainty might jeopardize the radiomics model prediction capabilities. To overcome at least part of this intrinsic limitation, the use of phantoms (i.e., an object's built-in shape and materials as close as possible to human tissue and organs) is a suitable means to assess and account for the possible similarities and differences. ${ }^{43}$ Radiomics features need also to be robust with respect to other possible sources of variability such as 
target volume motion, expansion, or shrinkage. To probe the feature resilience, test-retest approaches ${ }^{44,45}$ can be exploited to measure feature stability: for example, two datasets of images acquired at two different time points from the same subjects (e.g. patients or phantoms) or the use of cohorts from multiple sources. ${ }^{46,47}$ In this way, volatile or robust features can be identified and excluded from model development.

To ameliorate the reproducibility of radiomics features, several methods of harmonization have been proposed in the literature. The ComBat method initially developed for genomics aims to remove nonbiological differences related to scanner type in an effort to combine radiomics features extracted from data coming from different centers. ${ }^{32,48,49}$ Other methods include Neural Network training for radiomics feature standardization, ${ }^{50}$ intensity and diffusion maps harmonization, ${ }^{47,51}$ and data augmentation with generative adversarial networks (GAN). ${ }^{52}$ For a complete overview see. ${ }^{53}$

\section{2 | Feature extraction}

The essence of radiomics is the extraction of quantitative image features to characterize VOls. Hand-crafted radiomics features can be divided in five groups: size and shape-based features, descriptors of the image intensity histogram, descriptors of the (spatial) relationships between image voxels, features extracted from filtered images, and fractal features. ${ }^{54,55}$ Feature values are dependent on image preprocessing steps performed, such as filtering, or intensity discretization, and reconstruction. Furthermore, variations exist in feature nomenclature, mathematical definition, extraction methodology, and software implementation of the extraction algorithms. ${ }^{56-58}$ To harmonize radiomics features and model reports, all these differences has to be taken into account and clear specification included with each model. ${ }^{33}$

\section{3 | Exploratory analysis}

The true potential of radiomics approaches lies in the possibility to combine radiomics and other nonimage based features with the prediction endpoint to create a single data set. This approach allows the evaluation of possible correlations between features. However, some radiomics features that are highly correlated with other routine clinical features (such as tumor stage or age) might not provide additional meaningful information. Approaches, such as (unsupervised) clustering, PCA (Principal component analysis), ${ }^{59}$ or MRMR (maximum relevance minimum redundancy), ${ }^{1,60}$ identify and eliminate redundancy, for instance, by reducing highly correlated features to a single representative archetypical feature. This is a fundamental step to avoid overfitting. ${ }^{61,62}$ On the other hand, additional data collected, for example, from multiple segmentations or phantom studies can be used to test the feature robustness. ${ }^{63,64}$ This process of reduction should be described clearly, to avoid misinterpretation and aid in the unambiguous identification of relevant features. Also, univariable correlations of single radiomics features with clinical outcome is part of the exploratory analysis and could inform the subsequent modeling step, underlining relations between single radiomics features with clinical covariates of interest.

\section{4 | Modeling}

After features extraction and possible reduction, the creation of the radiomic model encompasses three major steps: feature selection, modeling methodology, and validation. Regarding the choice of modeling methodology, the identification of the best machine-learning method is a crucial step; thus, in an ideal scenario, multiple methods should be utilized and compared, ${ }^{65}$ and their implementation should be comprehensively documented. Another fundamental point in the modeling phase is the validation, which has to be performed to verify the applicability of the model in a real-world situation. Ideally, the model should be internally and externally validated, and the performance compared and clearly reported..$^{20,66,67}$ 


\subsection{1 | Feature selection}

The number of radiomics feature which could be extracted from medical images is technically unlimited. Several different filters, feature categories and other parameters can be used to mine the information hidden inside an imaging scan. Including all the possible features, even if practically possible, would result in overfitting which in turn renders the model useless for patients not previously evaluated (the so called curse of dimensionality). ${ }^{6,69}$ The most used approach is the reduction to archetypal feature representing a group or class of features, identified by dimensionality reduction techniques. Several different kinds of clustering algorithms and PCA are available and also this choice has to be justified and reported in detail, to promote transparency and replicability. Again, the same feature might be relevant for a given data set, segmented in a certain way for a specific end-point prediction but not important whit a different segmentation routine or a different cohort of patients.

\subsection{2 | Modeling methodology}

The choice of modeling technique has been proven to affect prediction performance in radiomics. ${ }^{65}$ Ideally, multiple modeling methodologies should be tested to select the best approach for the given data set and the other parameters involved in the creation of the model. Comparison between machine learning (ML) and deep learning (DL) approaches are common ${ }^{70,71}$ or even combination of both ${ }^{72}$ and the final choice has to take into account, along with the performance of the model, also the applicability of the proposed strategy in a real-world situation, considering for example computational burden or explainability of the resulting predictions. ${ }^{73,74}$ Another key point in the selection of modeling methodology is replicability by other researchers, in light of responsible and transparent research and innovation. This can be achieved, for example, by making the software code available in public repositories such as GitHub, ${ }^{75}$ Gitlab, ${ }^{76}$ and OpenML. ${ }^{77}$ Also, many scientific journals put in place, in the last years, tools to help data and algorithms sharing, making these available to the scientific community.

\subsection{3 | Validation}

Validation techniques are needed to assess the generalizability of the model predictions. Validation answer the question whether the model is predictive for the whole target patient population or just for a particular subset of cases analyzed. Model performances are typically measured in terms of discrimination and calibration. Discrimination is represented by the concordance statistics. For example the discrimination metric for the binary outcome is the receiver operating characteristic (ROC) curve, or area under the ROC curve (AUC). ${ }^{78} \mathrm{The} A U C$ is linked to the sensitivity and specificity of the model and represents the probability that a random patient matching an outcome is assigned in the class specific for that outcome with a larger probability than another random patient who does not match the outcome. The calibration, instead, is a measure of the agreement between observed outcomes and model predictions. ${ }^{79}$ Calibration can be reported using a calibration plot and calibration-in-thelarge/slope, with the Brier score, the mean squared prediction error, as a measure of overall performances.

The statistical methods used on both training and validation datasets need to be reported in detail. A valid model must exhibit statistical consistency between the training and validation sets. In terms of validation set selection, an externally validated model has more credibility than an internally validated one because validation with independent datasets is considered more robust. ${ }^{67,79-81}$ For "good radiomics practice", the reproducibility and replicability of the model should also be included in the validation step. Reproducibility relates to the verification of the result by independent researchers using the same methodology and data set, to verify the absence of errors, while replicability means the possibility of replicating the radiomics analysis with the same methodology but different appropriate datasets, to generalize the original findings. ${ }^{82-86}$ Reproducibility and replicability in radiomics 
are, however, not possible if researchers do not disclose all the details of the analysis performed. Each radiomics model must be accompanied by the of imaging protocol used for image collection, selected scans for analysis with exclusion and inclusion criteria, segmentations of VOls, detailed accounts of how features were extracted (including the preprocessing and feature reduction, and of the modeling methodology used (ideally, the code). ${ }^{87}$

\section{3 | THE RADIOMICS QUALITY SCORE: THREE YEARS LATER}

In 2017 the Radiomics Quality Score (RQS) was proposed in an effort to help the scientific community assessing the quality and scientific/clinical value of a radiomics study at a glance. ${ }^{4} \mathrm{~A}$ similar example is the Transparent Reporting of a multivariable prediction model for Individual Prognosis Or Diagnosis (TRIPOD) initiative. ${ }^{80}$ The RQS is determined by 16 key criteria which are assigned to a point value for a maximum of 36 points (100\%). These criteria cover image acquisition protocols, statistic data treatment, cohorts provenance and open science policies, encompassing all the relevant aspects which a reliable radiomics publication should present. Evaluating, 3 years after its publication, on what has been achieved with this initiative, we can clearly see that the road ahead is still long. The RQS was espoused with enthusiasm by the scientific community: however, was mainly used to assess the quality of already published studies. Recently, several systematic reviews have appeared, covering a variety of cancers: breast cancer, ${ }^{88,89}$ hepatocellular carcinoma, ${ }^{90}$ gliomas, ${ }^{91}$ prostate cancer, ${ }^{92}$ lung cancer, ${ }^{93,94}$ and renal carcinoma. ${ }^{95}$ All authors reported a very low RQS for the investigated publications (lower than 50\%, and in most cases lower than 20\%) with a lack of external validation, prospective study registration, and feature robustness test as the main causes. Also, open science policies and relevant clinical outcomes are among the most critical points raised for already published radiomics studies. To the best of our knowledge, however, researchers are still reticent in calculating and publishing the RQS for their newly developed radiomics models. In our opinion, this is slowing down considerably the acceptance of radiomics as a full-fledged ancillary method for critical clinical decision-making. The RQS should be seen as a quality seal of the published results more than a way of underlining the possible weaknesses of the proposed model. A low RQS score does not necessarily mean that the research is not sound but then it should be part of the discussion and motivate the authors to carefully think through all the steps performed and discuss why deviation from "good practice" is justified or what can be done better. Editors, reviewers, and readers should be able to ascertain whether a radiomic study is compliant with good radiomics practice or, alternatively, whether the authors have justified any noncompliance. Overly optimistic claims concerning robustness and generalizability diminish the scientific and clinical impact and should be avoided.

The consideration of the apparently limited impact of the RQS thus far might spur the scientific community to ask the question of whether the RQS requires regular evaluation and updating in such a fast-evolving field, like radiomics. For example, RQS was tailored on hand-crafted features while nowadays deep learning is gaining momentum and some of its inherent strengths and weaknesses might not be captured correctly in the current version of the RQS score.

\section{4 | RADIOMICS TOWARDS PERSONALIZED MEDICINE}

\section{1 | Virtual biopsy}

In patients with cancer, different parts of the tumor have distinct molecular characteristics, but also different lesions (metastases) from a tumor disease, which may have a role in terms of therapeutic efficacy, and such differences might change over time. As it is not possible to take samples of every part of each tumor at multiple time points, the optimal characterization of tumors is not achieved using biopsy. ${ }^{96}$ However, radiomics might be used to "sample" different parts of the tumor at different time points (i.e., different scans) and, along with genomic data, used as a virtual biopsy tool. ${ }^{97,98}$ The combination of radiomics and genomics is called radiogenomics and has 
gathered considerable attention in the last years, as a way of augmenting the power of both approaches, for personalized medicine and treatment follow-up. ${ }^{99-101}$

\section{2 | Beyond oncology}

Radiomics was mostly employed in oncology up to now, but in the last years showed its potential for other clinical applications. Radiomics analysis was performed on MRI scans to distinguish between different cognitive disorders such as Alzheimer's disease, ${ }^{102-104}$ autism spectrum disorder ${ }^{105}$ or amnestic mild cognitive impairment (aMCl). ${ }^{106,107}$ Another field in which radiomics might give a relevant contribution is bone disease study. Radiomics methods have been reported for the early identification of osteoporosis ${ }^{108}$ or for classification of osteoporotic patients compared to normal subjects or suffering from osteopenia. ${ }^{109}$ Radiomics was also applied to maxillofacial radiology. ${ }^{110}$ Among the new application of radiomics, 2020 brought forth a clear winner. The outbreak of the new SARS-CoV-2 virus and the subsequent pandemic placed the research community under unprecedented pressure in the race to find better diagnostic and therapeutic tools to fight this threat to human health. The correlation between COVID-19 infection and lung CT scans characteristics was reported early during the year, ${ }^{111,112}$ suggesting that radiomics might be a successful approach in the early diagnosis and prognosis of COVID-19 patients. In the last months of 2020, the scientific community has produced a deluge of radiomics publications related to COVID-19. Radiomics has been used for diagnosis, ${ }^{41,113,114}$ to distinguishing pulmonary infections from different sources ${ }^{115-117}$ or predicting the length of hospitalization. ${ }^{118}$ A complete overview of all the radiomics approaches for COVID-19 is beyond the scope of the present review; a relevant collection of the most interesting articles can be found in. ${ }^{119,120}$

\subsection{Delta radiomics}

The vast majority of radiomics methods published focus on imaging data acquired at a single time point, mostly imaging tumors before the start of treatment. Delta-radiomics introduces a time component with the extraction of quantitative features from image sets acquired over the course of treatment, ${ }^{121-123}$ which provides information on the evolution of feature values. Delta-radiomics promises to improve diagnosis, prognosis, prediction, monitoring, image-based intervention, or assessment of therapeutic response. ${ }^{124,125}$ Delta radiomics has been proven effective in the study of immunotherapy response ${ }^{126,127}$ or to predict recurrence in oncological patients. ${ }^{128}$

\subsection{Open science and data sharing}

There is a pressing need to embrace knowledge and data-sharing technology, ${ }^{129}$ which transcends institutional and national boundaries. ${ }^{130}$ This is especially true for radiomics whose potency is directly linked to the amount and quality of data available. Larger datasets, deeper clinical and molecular information and homogeneous imaging sources will result in more robust and reliable radiomics models. To unlock the full potential of radiomics for clinical decisionmaking, the research and clinical community must strive for truly open science-sharing datasets, algorithms, best practices, and finding new ways to improve collaborations. One initiative to accomplish these goals is CancerLinQ, ${ }^{131}$ the ASCO data centralization approach. Other initiatives are WorldCAT and its European counterpart euroCAT ${ }^{132}$ that $^{2}$ consist of a novel data-federated approach that successfully links radiotherapy institutes in the Netherlands, Germany, Belgium, Italy, Denmark, Australia, China, India, South Africa, Ireland, UK, USA, and Canada. ${ }^{133,134}$ Other important initiatives include The Cancer Imaging Archive (TCIA), ${ }^{135}$ The Quantitative Imaging Network (QIN), ${ }^{136}$ the Quantitative Imaging Biomarkers Alliance (QIBA), ${ }^{137}$ the MEDomics consortium, ${ }^{138}$ and Quantitative Imaging in Cancer: Connecting Cellular Processes with Therapy (QulC-ConCePT). ${ }^{139}$ To overcome data-sharing issues, such as privacy concerns or 
insufficient infrastructures, an approach based on distributed machine learning for radiomics model's creation has been proposed. The concept of distributed (federated, privacy-preserving) machine learning is not new in healthcare application $^{8,133}$ but has recently shown its potential for radiomics. ${ }^{140,141}$ For example, Shi et al. ${ }^{142}$ performed a multi-center study to develop a radiomic signature for lung cancer in one institution and validated the performance in an independent institution, without the need for data exchange. In another recent case study, Bogowicz et al. ${ }^{143}$ developed and validated a radiomic signature for head and neck cancer, training the model remotely from six independent cohorts, showing that the performances of the distributed model were as good as the one obtained with traditional radiomic approach. The next step in this open science initiative for radiomics should be the creation of database to store and cross-reference radiomics features and relevant clinical data (radiomics ontology ${ }^{144,145}$ ). Also, the accessibility of radiomics, in general, must be improved and some initiatives in this regard are already in place, especially from a software perspective. Several open-source or freeware softwares are already available ${ }^{58,146-148}$ and code sharing is becoming more and more accepted in the scientific community.

\subsection{New applications of radiomics}

In the last years, radiomics had broadened its horizon, pushing the boundaries of what was achieved thus far. The possibility to use radiomics signatures to explore new medical conditions is expanding. Recently, a signature for the determination of chromosome deletion in low-grade glioma (LGG) patients ${ }^{149}$ has been reported. The authors developed two radiomics signatures, composed of seven and five features respectively, extracted from T2- and T1weighted post-contrast MRI. Both signatures showed an accuracy higher than 0.70 . Another recent example of the novel pathways for radiomics is represented by the paper of $\mathrm{Mu}$ et al. ${ }^{150}$ The authors realized a radiomics signature on baseline PET-CT image of NSCLC patients treated with immune checkpoint inhibitors. The signature was able to predict prolonged weight loss syndrome (cachexia), which contributes to primary resistance to immune checkpoint inhibitors therapy. The signature was also able to predict durable clinical benefit (DCB), progressionfree survival (PFS), and overall survival (OS). The performance on this signature in the external testing cohort was satisfying (AUC higher than 0.65). Radiomics was also used to explore different imaging techniques such as ultrasound imaging (US). Chiappa et al. ${ }^{151}$ developed a signature for differential diagnosis of myometrial tumors. While the study is based on single-center data, the performance of the signature in discriminating between patients with sarcoma or myoma was very good (accuracy of 0.85), proving that also US imaging can be successfully mined via radiomics approaches. The combination of radiomics and digital pathology has been also recently reported for different kinds of cancer such as nasopharyngeal carcinoma, ${ }^{152}$ non-small cell lung cancer, ${ }^{153}$ and pancreatic cancer ${ }^{154}$ among others. All these examples showcase the versatility of radiomics which could stem from a closer collaboration with clinical experts and Al scientists beyond the current applications.

\section{5 | CONCLUSIONS AND FUTURE OUTLOOK}

The outlook for radiomics is very promising and the efforts devoted to its standardization are already bearing their fruits. However, there is still much work to do, especially to link fundamental research to current clinical practice. Physicians and healthcare personnel should be involved from the start of the process, along with relevant authorities. On the other hand, more effort should be devoted in the technological transfer, taking the published research and perform the necessary steps to bring it from a (validated) proof-of-concept to the clinic. This also emphasizes the need for comprehensive and universal indicators (such as the RQS) of the quality of a model. The normative framework is currently evolving along with the innovations in the field of Al-driven healthcare. For example, FDA is gathering feedbacks and propositions to draft a novel regulatory framework for Al/ML-based medical devices. ${ }^{155}$ Paradigms need to re-invented to allow these breakthroughs to reach the clinic in the very near 
future, always putting patient's welfare first. Personalized, patient-centric medicine is almost a reality and radiomics is playing a major role in it and will represent one of the key factors for the future of healthcare.

\section{ACKNOWLEDGMENTS}

Authors acknowledge financial support from ERC advanced grant (ERC-ADG-2015 n 694812 - Hypoximmuno), ERC-2018-PoC: 813200-CL-IO, ERC-2020-PoC: 957565-AUTO. DISTINCT) Authors also acknowledge financial support from SME Phase 2 (RAIL n673780), the European Union's Horizon 2020 research and innovation program under grant agreement: ImmunoSABR $n^{\circ}$ 733008, MSCA-ITN-PREDICT n 766276, CHAIMELEON n 952172 , EuCanlmage $n^{\circ}$ 952103, TRANSCAN Joint Transnational Call 2016 (JTC2016 CLEARLY n UM 2017-8295) and Interreg V-A Euregio Meuse-Rhine (EURADIOMICS n EMR4).

\section{CONFLICT OF INTERESTS}

The authors declare that there are no conflict of interests.

\section{ORCID}

Fabio Bottari (D) https://orcid.org/0000-0003-0785-3412

Ralph T.H. Leijenaar (D) http://orcid.org/0000-0001-8642-9657

\section{REFERENCES}

1. Aerts HJ, Velazquez ER, Leijenaar RT, et al. Decoding tumour phenotype by noninvasive imaging using a quantitative radiomics approach. Nat Commun. 2014;5:4006.

2. Hood L, Friend SH. Predictive, personalized, preventive, participatory (P4) cancer medicine. Nat Rev Clin Oncol. 2011;8(3):184-187.

3. Lambin P, Rios-Velazquez E, Leijenaar R, et al. Radiomics: extracting more information from medical images using advanced feature analysis. Eur J Cancer. 2012;48(4):441-446.

4. Lambin P, Leijenaar RTH, Deist TM, et al. Radiomics: the bridge between medical imaging and personalized medicine. Nat Rev Clin Oncol. 2017;14(12):749-762.

5. Kumar V, Gu Y, Basu S, et al. Radiomics: the process and the challenges. Magn Reson Imaging. 2012;30(9):1234-1248.

6. AA.VV. Medicine: computers by the bedside. Nature. 1969;224(5220):636-637.

7. Schoolman HM, Bernstein LM. Computer use in diagnosis, prognosis, and therapy. Science (80-). 1978;200(4344): 926 LP-926931.

8. Zerka F, Barakat S, Walsh S, et al. Systematic review of privacy-preserving distributed machine learning from federated databases in health care. JCO Clin Cancer Informatics. 2020;4:184-200.

9. Gillies RJ, Kinahan PE, Hricak H. Radiomics: images are more than pictures, they are data. Radiology. 2016;278(2): 563-577. https://doi.org/10.1148/radiol.2015151169

10. Liu Z, Wang S, Dong D, et al. The applications of radiomics in precision diagnosis and treatment of oncology: Opportunities and challenges. Theranostics. 2019;9(5):1303-1322.

11. Zheng B-H, Liu L-Z, Zhang Z-Z, et al. Radiomics score: a potential prognostic imaging feature for postoperative survival of solitary HCC patients. BMC Cancer. 2018;18(1):1148.

12. Monti S, Aiello M, Incoronato M, et al. DCE-MRI pharmacokinetic-based phenotyping of invasive ductal carcinoma: a radiomic study for prediction of histological outcomes. Contrast Media Mol Imaging. 2018;2018:5076269.

13. Bibault JE, Giraud P, Housset M, et al. Deep Learning and Radiomics predict complete response after neo-adjuvant chemoradiation for locally advanced rectal cancer. Sci Rep. 2018;8(1):1-8.

14. Song Xli, Ren JL, Zhao D, Wang L, Ren H, Niu J. Radiomics derived from dynamic contrast-enhanced MRI pharmacokinetic protocol features: the value of precision diagnosis ovarian neoplasms. Eur Radiol. 2020;I.

15. Roelofs E, Dekker A, Meldolesi E, Stiphout RGPM, Valentini V, Lambin P. International data-sharing for radiotherapy research: an open-source based infrastructure for multicentric clinical data mining. Radiother Oncol. 2014;110(2): 370-374.

16. Roelofs E, Persoon L, Nijsten S, Wiessler W, Dekker A, Lambin P. Benefits of a clinical data warehouse with data mining tools to collect data for a radiotherapy trial. Radiother Oncol. 2013;108(1):174-179.

17. Miotto R, Li L, Kidd BA, Dudley JT. Deep patient: an unsupervised representation to predict the future of patients from the electronic health records. Sci Rep. 2016;6(1):26094. 
18. Nead KT, Gaskin G, Chester C, et al. Androgen deprivation therapy and future Alzheimer's disease risk. J Clin Oncol. 2015;34(6):566-571.

19. Gatenby RA, Grove O, Gillies RJ. Quantitative imaging in cancer evolution and ecology. Radiology. 2013;269(1):8-14.

20. Lambin P, Zindler J, Vanneste BG, et al. Decision support systems for personalized and participative radiation oncology. Adv Drug Deliv Rev. 2017;109:131-153.

21. Vickers AJ. Prediction models: revolutionary in principle, but do they do more good than harm? J Clin Oncol. 2011; 29(22):2951-2952.

22. Limkin EJ, Sun R, Dercle L, et al. Promises and challenges for the implementation of computational medical imaging (radiomics) in oncology. Ann Oncol. 2017;28(6):1191-1206.

23. Leijenaar RT, Bogowicz M, Jochems A, et al. Development and validation of a radiomic signature to predict HPV (p16) status from standard CT imaging: A multicenter study. Br J Radiol. 2018;91(1086):20170498.

24. Leite AF, Vasconcelos K, de F, Willems H, Jacobs R. Radiomics and machine learning in oral healthcare. Proteomics Clin Appl. 2020;14(3):1900040.

25. Sun $\mathrm{H}$, Chen $\mathrm{Y}$, Huang Q, et al. Psychoradiologic utility of MR imaging for diagnosis of attention deficit hyperactivity disorder: a radiomics analysis. Radiology. 2018;287(2):620-630.

26. Lovinfosse P, Visvikis D, Hustinx R, Hatt M. FDG PET radiomics: a review of the methodological aspects. Clin Transl Imaging. 2018;6(5):379-391. https://doi.org/10.1007/s40336-018-0292-9

27. Sibille L, Seifert R, Avramovic N, et al. 18F-FDG PET/CT uptake classification in lymphoma and lung cancer by using deep convolutional neural networks. Radiology. 2020;294(2):445-452. https://doi.org/10.1148/radiol.2019191114

28. Röhrich S, Hofmanninger J, Prayer F, Müller H, Prosch H, Langs G. Prospects and challenges of radiomics by using nononcologic routine chest CT. Radiol Cardiothorac Imaging. 2020;2(4):e190190.

29. Bogowicz M, Leijenaar RTH, Tanadini-Lang S, et al. Post-radiochemotherapy PET radiomics in head and neck cancer The influence of radiomics implementation on the reproducibility of local control tumor models. Radiother Oncol. 2017; 125(3):385-391.

30. Timmeren JE, Leijenaar RTH, Elmpt W, et al. Survival prediction of non-small cell lung cancer patients using radiomics analyses of cone-beam CT images. Radiother Oncol. 2017;123(3):363-369.

31. Yip SSF, Aerts HJWL. Applications and limitations of radiomics. Phys Med Biol. 2016;61(13):R150-R166.

32. Orlhac F, Frouin F, Nioche C, Ayache N, Buvat I. Validation of a method to compensate multicenter effects affecting CT radiomics. Radiology. 2019;291(1):53-59.

33. Zwanenburg $A$, Vallières $M$, Abdalah MA, et al. The image biomarker standardization initiative: Standardized quantitative radiomics for high-throughput image-based phenotyping. Radiology. 2020;295(2):328-338.

34. Polan DF, Brady SL, Kaufman RA. Tissue segmentation of computed tomography images using a Random Forest algorithm: a feasibility study. Phys Med Biol. 2016;61(17):6553-6569.

35. Porz N, Bauer S, Pica A, et al. Multi-modal glioblastoma segmentation: man versus machine. PLoS One. 2014;9(5):1-9.

36. Porz N, Habegger S, Meier R, et al. Fully automated enhanced tumor compartmentalization: man vs. machine reloaded. PLoS One. 2016;11(11):1-16.

37. Balagurunathan Y, Gu Y, Wang H, et al. Reproducibility and prognosis of quantitative features extracted from CT images. Transl Oncol. 2014;7(1):72-87.

38. Grootjans W, Tixier F, Vos CS, et al. The impact of optimal respiratory gating and image noise on evaluation of intratumor heterogeneity on 18F-FDG PET imaging of lung cancer. J Nucl Med. 2016;57(11):1692-1698.

39. Zwanenburg A, Leger S, Agolli L, et al. Assessing robustness of radiomic features by image perturbation. Sci Rep. 2019;9(1):614.

40. Farag AA, Munim HEAE, Graham JH, Farag AA. A novel approach for lung nodules segmentation in chest CT using level sets. IEEE Trans Image Process. 2013;22(12):5202-5213.

41. Guiot J, Vaidyanathan A, Deprez L, et al. Development and validation of an automated radiomic CT signature for detecting COVID-19. Diagnostics. 2021;11(1):41. https://doi.org/10.3390/diagnostics11010041

42. Mackin D, Fave $X$, Zhang L, et al. Measuring Computed tomography scanner variability of radiomics features. Invest Radiol. 2015;50(11):757-765.

43. Berenguer R, Pastor-Juan M, Canales-Vázquez J, et al. Radiomics of CT features may be nonreproducible and redundant: Influence of CT acquisition parameters. Radiology. 2018;288(2):407-415.

44. Zhao B, Tan Y, Tsai W-Y, et al. Reproducibility of radiomics for deciphering tumor phenotype with imaging. Sci Rep. 2016;6(1):23428.

45. Haarburger C, Müller-Franzes G, Weninger L, Kuhl C, Truhn D, Merhof D. Radiomics feature reproducibility under inter-rater variability in segmentations of CT images. Sci Rep. 2020;10(1):1-10.

46. Timmeren JE, Leijenaar RTH, Elmpt W, et al. Test-retest data for radiomics feature stability analysis: generalizable or study-specific? Tomogr (Ann Arbor, Mich). 2016;2(4):361-365. 
47. Peerlings J, Woodruff HC, Winfield JM, et al. Stability of radiomics features in apparent diffusion coefficient maps from a multi-centre test-retest trial. Sci Rep. 2019;9(1):4800.

48. Da-ano R, Masson I, Lucia F, et al. Performance comparison of modified ComBat for harmonization of radiomic features for multicenter studies. Sci Rep. 2020;10(1):1-12.

49. Orlhac F, Boughdad S, Philippe C, et al. A postreconstruction harmonization method for multicenter radiomic studies in PET. J Nucl Med. 2018;59(8):1321-1328.

50. Andrearczyk V, Depeursinge A, Müller H. Neural network training for cross-protocol radiomic feature standardization in computed tomography. J Med Imaging. 2019;6(02):1.

51. Crombé A, Kind M, Fadli D, et al. Intensity harmonization techniques influence radiomics features and radiomicsbased predictions in sarcoma patients. Sci Rep. 2020;10(1):1-13.

52. Sandfort V, Yan K, Pickhardt PJ, Summers RM. Data augmentation using generative adversarial networks (CycleGAN) to improve generalizability in CT segmentation tasks. Sci Rep. 2019;9(1):1-9.

53. Da-Ano R, Visvikis D, Hatt M. Harmonization strategies for multicenter radiomics investigations. Phys Med Biol. 2020;65(24):24TR02.

54. Ranjbar S, Ross Mitchell J Chapter 8 - An Introduction to Radiomics: An Evolving Cornerstone of Precision Medicine. In: Depeursinge A, S. Al-Kadi O, Mitchell JRBT-BTA, eds. Academic Press; 2017:223-245. https://doi.org/10. 1016/B978-0-12-812133-7.00008-9

55. Antonelli L, Guarracino MR, Maddalena L, Sangiovanni M. Integrating imaging and omics data: a review. Biomed Signal Process Control. 2019;52:264-280. https://doi.org/10.1016/j.bspc.2019.04.032

56. Hatt M, Tixier F, Pierce L, Kinahan PE, Le Rest CC, Visvikis D. Characterization of PET/CT images using texture analysis: the past, the present... any future? Eur J Nucl Med Mol Imaging. 2017;44(1):151-165.

57. Fang YH, Lin C-Y, Shih M-J, et al. Development and evaluation of an open-source software package "CGITA" for quantifying tumor heterogeneity with molecular images. BioMed Res Int. 2014;2014:248505.

58. Zhang L, Fried DV, Fave XJ, Hunter LA, Yang J, Court LE. IBEX: an open infrastructure software platform to facilitate collaborative work in radiomics. Med Phys. 2015;42(3):1341-1353.

59. Delzell DAP, Magnuson S, Peter T, Smith M, Smith BJ. Machine Learning and feature selection methods for disease classification with application to lung cancer screening image data. Front Oncol. 2019;9:1393.

60. Radovic M, Ghalwash M, Filipovic N, Obradovic Z. Minimum redundancy maximum relevance feature selection approach for temporal gene expression data. BMC Bioinformatics. 2017;18(1):9.

61. Ying Xue. An overview of overfitting and its solutions. J Phys Conf Ser. 2019;1168. https://doi.org/10.1088/1742$6596 / 1168 / 2 / 022022$

62. Song H, Kim M, Park D, Lee J-G Prestopping: how does early stopping help generalization against label noise? arXiv:1911.08059.

63. Lee $\mathrm{S}-\mathrm{H}$, Cho H, Lee HY, Park H. Clinical impact of variability on CT radiomics and suggestions for suitable feature selection: a focus on lung cancer. Cancer Imaging. 2019;19(1):54.

64. Qiu Q, Duan J, Duan Z. Reproducibility and non-redundancy of radiomic features extracted from arterial phase CT scans in hepatocellular carcinoma patients: impact of tumor segmentation variability. Quant Imaging Med Surg. 2019;9(3).

65. Parmar C, Grossmann P, Bussink J, Lambin P, Aerts HJWL. Machine learning methods for quantitative radiomic biomarkers. Sci Rep. 2015;5(1):13087.

66. Garau N, Paganelli C, Summers P, et al. External validation of radiomics-based predictive models in low-dose CT screening for early lung cancer diagnosis. Med Phys. 2020;47(9):4125-4136.

67. Debray TPA, Vergouwe Y, Koffijberg H, Nieboer D, Steyerberg EW, Moons KGM. A new framework to enhance the interpretation of external validation studies of clinical prediction models. J Clin Epidemiol. 2015;68(3):279-289.

68. Park JE, Park SY, Kim HJ, Kim HS. Reproducibility and generalizability in radiomics modeling: possible strategies in radiologic and statistical perspectives. Korean J Radiol. 2019;20(7):1124-1137.

69. Altman N, Krzywinski M. The curse(s) of dimensionality. Nat Methods. 2018;15(6):399-400.

70. Capobianco E, Deng J. Radiomics at a glance: a few lessons learned from learning approaches. Cancers. 2020;12(9). https://doi.org/10.3390/cancers12092453

71. Avanzo M, Wei L, Stancanello J, et al. Machine and deep learning methods for radiomics. Med Phys. 2020;47(5):e185-e202.

72. Liu Q-P, Xu X, Zhu F-P, Zhang Y-D, Liu X-S. Prediction of prognostic risk factors in hepatocellular carcinoma with transarterial chemoembolization using multi-modal multi-task deep learning. EClinicalMedicine. 2020;23:23.

73. Giraud P, Giraud P, Gasnier A, et al. Radiomics and machine learning for radiotherapy in head and neck cancers. Front Oncol. 2019;9:174.

74. Choy G, Khalilzadeh O, Michalski M, et al. Current applications and future impact of machine learning in radiology. Radiology. 2018;288(2):318-328.

75. https://github.com/ 
76. https://about.gitlab.com/

77. https://www.openml.org/

78. DeLong ER, DeLong DM, Clarke-Pearson DL. Comparing the areas under two or more correlated receiver operating characteristic curves: a nonparametric approach. Biometrics. 1988;44(3):837-845.

79. Steyerberg EW, Vickers AJ, Cook NR, et al. Assessing the performance of prediction models. Epidemiology. 2010; 21(1):128-138. https://doi.org/10.1097/ede.0b013e3181c30fb2

80. Collins GS, Reitsma JB, Altman DG, Moons KGM. Transparent reporting of a multivariable prediction model for individual prognosis or diagnosis (TRIPOD): the TRIPOD Statement. BMC Med. 2015;13(1):1.

81. Lemeshow S, Hosmer DW Jr. A review of goodness of fit statistics for use in the development of logistic regression models1. Am J Epidemiol. 1982;115(1):92-106.

82. Leek JT, Peng RD. Statistics: P values are just the tip of the iceberg. Nature. 2015;520(7549):612.

83. Drummond C Replicability is not reproducibility: nor is it good science. Proc Eval Methods Mach Learn Work 26th ICML, Montr Canada, 2009. Published online 2009.

84. Peng RD. Reproducible research in computational science. Science. 2011;334(6060):1226-1227. https://doi.org/10. 1126/science.1213847

85. Peng RD, Dominici F, Zeger SL. Reproducible epidemiologic research. Am J Epidemiol. 2006;163(9):783-789.

86. Fiset S, Welch ML, Weiss J, et al. Repeatability and reproducibility of MRI-based radiomic features in cervical cancer. Radiother Oncol. 2019;135:107-114.

87. Kalendralis $\mathrm{P}$, Shi Z, Traverso A, et al. FAIR-compliant clinical, radiomics and DICOM metadata of RIDER, interobserver, Lung1 and head-Neck1 TCIA collections. Med Phys. 2020;47(11):5931-5940.

88. Valdora F, Houssami N, Rossi F, Calabrese M, Tagliafico AS. Rapid review: radiomics and breast cancer. Breast Cancer Res Treat. 2018;169(2):217-229.

89. Granzier RWY, Nijnatten TJA, Woodruff HC, Smidt ML, Lobbes MBI. Exploring breast cancer response prediction to neoadjuvant systemic therapy using MRI-based radiomics: a systematic review. Eur J Radiol. 2019;121:108736.

90. Wakabayashi T, Ouhmich F, Gonzalez-Cabrera C, et al. Radiomics in hepatocellular carcinoma: a quantitative review. Hepatol Int. 2019;13(5):546-559.

91. Park JE, Kim HS, Kim D, et al. A systematic review reporting quality of radiomics research in neuro-oncology: Toward clinical utility and quality improvement using high-dimensional imaging features. BMC Cancer. 2020;20(1):1-11.

92. Stanzione A, Gambardella M, Cuocolo R, Ponsiglione A, Romeo V, Imbriaco M. Prostate MRI radiomics: a systematic review and radiomic quality score assessment. Eur J Radiol. 2020;129:109095.

93. Chetan MR, Gleeson FV. Radiomics in predicting treatment response in non-small-cell lung cancer: current status, challenges and future perspectives. Eur Radiol. 2020;31:1049-1058.

94. Abdurixiti M, Nijiati M, Shen R, Ya Q, Abuduxiku N, Nijiati M. Current progress and quality of radiomic studies for predicting EGFR mutation in patients with non-small cell lung cancer using PET/CT images: a systematic review. $\mathrm{Br}$ J Radiol. 2021;94:20201272.

95. Ursprung S, Beer L, Bruining A, et al. Radiomics of computed tomography and magnetic resonance imaging in renal cell carcinoma-a systematic review and meta-analysis. Eur Radiol. 2020;30(6):3558-3566.

96. Banna GL, Olivier T, Rundo F, et al. The Promise of digital biopsy for the prediction of tumor molecular features and clinical outcomes associated with immunotherapy. Front Med. 2019;6:172.

97. Martin-Gonzalez P, Crispin-Ortuzar M, Rundo L, et al. Integrative radiogenomics for virtual biopsy and treatment monitoring in ovarian cancer. Insights Imaging. 2020;11(1):94.

98. Shofty B, Artzi M, Shtrozberg S, et al. Virtual biopsy using MRI radiomics for prediction of BRAF status in melanoma brain metastasis. Sci Rep. 2020;10(1):1-7.

99. Limkin EJ, Sun R. Radiomics to predict response to immunotherapy: an imminent reality? Future Oncol. 2020. 2020; 16(23):1673-1676.

100. Tselikas L, Sun R, Ammari S, et al. Role of image-guided biopsy and radiomics in the age of precision medicine. Chinese Clin Oncol. 2019;8(6):6-13.

101. Ismail M, Correa R, Bera K, et al. Spatial-And-Context aware (SpACe) "virtual biopsy" radiogenomic maps to target tumor mutational status on structural MRI. Published online 2020:1-10. http://arxiv.org/abs/2006.09878

102. Ranjbar S, Velgos SN, Dueck AC, Geda YE, Mitchell JR. Brain MR radiomics to differentiate cognitive disorders. J Neuropsychiatry Clin Neurosci. 2019;31(3):210-219.

103. Zhou H, Jiang J, Lu J, Wang M, Zhang H, Zuo C. Dual-model radiomic biomarkers predict development of mild cognitive impairment progression to Alzheimer's disease. Front Neurosci. 2019;12. https://doi.org/10.3389/fnins. 2018.01045

104. Zhao K, Ding Y, Han Y, et al. Independent and reproducible hippocampal radiomic biomarkers for multisite Alzheimer's disease: diagnosis, longitudinal progress and biological basis. Sci Bull. 2020;65(13):1103-1113. 
105. Chaddad A, Desrosiers C, Hassan L, Tanougast C. Hippocampus and amygdala radiomic biomarkers for the study of autism spectrum disorder. BMC Neurosci. 2017;18:1-12.

106. Feng Q, Song Q, Wang M, et al. Hippocampus radiomic biomarkers for the diagnosis of amnestic mild cognitive impairment: a machine learning method. Front Aging Neurosci. 2019;11:1-10.

107. Li Y, Jiang J, Lu J, Jiang J, Zhang H, Zuo C. Radiomics: a novel feature extraction method for brain neuron degeneration disease using 18F-FDG PET imaging and its implementation for Alzheimer's disease and mild cognitive impairment. Ther Adv Neurol Disord. 2019;12. https://doi.org/10.1177/1756286419838682

108. He L, Liu Z, Liu C, et al. Radiomics based on lumbar spine magnetic resonance imaging to detect osteoporosis. Acad Radiol. 2021;28(6):e165-e171. https://doi.org/10.1016/j.acra.2020.03.046

109. Rastegar S, Vaziri M, Qasempour Y, et al. Radiomics for classification of bone mineral loss: a machine learning study. Diagn Interv Imaging. 2020;101(9):599-610.

110. Bianchi J, Gonçalves JR, Ruellas A, et al. Software comparison to analyze bone radiomics from high resolution CBCT scans of mandibular condyles. Dentomaxillofacial Radiol. 2019;48(6):1-10.

111. Wang H, Wei R, Rao G, Zhu J, Song B, Characteristic CT. findings distinguishing 2019 novel coronavirus disease (COVID-19) from influenza pneumonia. Eur Radiol. 2020;30(9):4910-4917.

112. Revel M-P, Parkar AP, Prosch H, et al. COVID-19 patients and the radiology department - advice from the European Society of Radiology (ESR) and the European Society of Thoracic Imaging (ESTI). Eur Radiol. 2020;30:1-7.

113. Guiot J, Vaidyanathan A, Deprez L. Development and validation of an automated radiomic CT signature for detecting COVID-19. medRxiv. 2020. https://doi.org/10.1101/2020.04.28.20082966

114. Li L, Qin L, Xu Z, et al. Using artificial intelligence to detect COVID-19 and Community-acquired pneumonia based on pulmonary CT: evaluation of the diagnostic accuracy. Radiology. 2020;296(2):E65-E71.

115. Zeng QQ, Zheng $\mathrm{KI}$, Chen J, et al. Radiomics-based model for accurately distinguishing between severe acute respiratory syndrome associated coronavirus 2 (SARS-CoV-2) and influenza A infected pneumonia. MedComm. 2020; June:1-9. https://doi.org/10.1002/mco2.14

116. Chen $\mathrm{Y}$, Wang $\mathrm{Y}$, Zhang $\mathrm{Y}$, et al. A quantitative and radiomics approach to monitoring ards in COVID-19 patients based on chest CT: A retrospective cohort study. Int J Med Sci. 2020;17(12):1773-1782.

117. Bai HX, Wang R, Xiong Z, et al. Artificial Intelligence augmentation of radiologist performance in distinguishing COVID-19 from pneumonia of other origin at chest CT. Radiology. 2020;296(3):E156-E165.

118. Yue H, Yu Q, Liu C, et al. Machine learning-based CT radiomics method for predicting hospital stay in patients with pneumonia associated with SARS-CoV-2 infection: a multicenter study. Ann Transl Med. 2020;8(14):859.

119. Shi F, Wang J, Shi J, et al. Review of artificial intelligence techniques in imaging data acquisition, segmentation and diagnosis for COVID-19. IEEE Rev Biomed Eng. 2021;14:4-15. https://doi.org/10.1109/rbme.2020.2987975

120. Kundu S, Elhalawani H, Gichoya JW, Kahn CE. How might Al and chest imaging help unravel COVID-19's mysteries? Radiol Artif Intell. 2020;2(3):e200053.

121. Fave X, Zhang L, Yang J, et al. Delta-radiomics features for the prediction of patient outcomes in non-small cell lung cancer. Sci Rep. 2017;7(1):588.

122. Ma Y, Ma W, Xu X, Cao F. How does the delta-radiomics better differentiate pre-invasive GGNs from invasive GGNs? Front Oncol. 2020;10(July):1-7.

123. Chen H, Shi L, Nguyen KNB, et al. MRI Radiomics for Prediction of Tumor Response and Downstaging in Rectal Cancer Patients after Preoperative Chemoradiation. Adv Radiat Oncol. 2020;5:1-10.

124. Nasief H, Zheng C, Schott D, et al. A machine learning based delta-radiomics process for early prediction of treatment response of pancreatic cancer. npj Precis Oncol. 2019;3(1):1-10.

125. Lin P, Yang PF, Chen S, et al. A delta-radiomics model for preoperative evaluation of neoadjuvant chemotherapy response in high-grade osteosarcoma. Cancer Imaging. 2020;20(1):1-12.

126. Liu Y, Wu M, Zhang Y, et al. Imaging Biomarkers to Predict and Evaluate the Effectiveness of Immunotherapy in Advanced Non-Small-Cell Lung Cancer. Front Oncol. 2021;11:773.

127. Colen RR, Rolfo C, Ak M, et al. Radiomics analysis for predicting pembrolizumab response in patients with advanced rare cancers. J Immunother Cancer. 2021;9(4):e001752.

128. Fatima K, Dasgupta A, DiCenzo D, et al. Ultrasound delta-radiomics during radiotherapy to predict recurrence in patients with head and neck squamous cell carcinoma. Clin Transl Radiat Oncol. 2021;28:62-70.

129. Deasy JO, Bentzen SM, Jackson A, et al. Improving normal tissue complication probability models: the need to adopt a "data-pooling" culture. Int J Radiat Oncol Biol Phys. 2010;76(3):S151-S154. https://doi.org/10.1016/j.jirobp.2009.06.094

130. Skripcak T, Belka C, Bosch W, et al. Creating a data exchange strategy for radiotherapy research: towards federated databases and anonymised public datasets. Radiother Oncol J Eur Soc Ther Radiol Oncol. 2014;113(3):303-309.

131. Schilsky RL, Michels DL, Kearbey AH, Yu PP, Hudis CA. Building a rapid learning health care system for oncology: the regulatory framework of CancerLinQ. J Clin Oncol Off J Am Soc Clin Oncol. 2014;32(22):2373-2379.

132. http://www.eurocat.info/ 
133. Deist TM, Jochems A, Soest J, et al. Infrastructure and distributed learning methodology for privacy-preserving multi-centric rapid learning health care: euroCAT. Clin Transl Radiat Oncol. 2017;4:24-31.

134. Lambin P, Roelofs E, Reymen B, et al. 'Rapid Learning health care in oncology' - An approach towards decision support systems enabling customised radiotherapy'. Radiother Oncol. 2013;109(1):159-164.

135. The Cancer Imaging Archive. TCIA Collections. cancerimagingarchive.net. Published 2017. https://www. cancerimagingarchive.net/

136. National Cancer Institute. Quantitative Imaging Network (QIN). Published 2017. https://imaging.cancer.gov/ programs_resources/specialized_initiatives/qin.htm

137. Radiological Society of North America. Quantitative Imaging Biomarkers Alliance® (QIBA®). Published 2017. https://www.rsna.org/qiba/

138. www.medomics.ai

139. QuiC ConCePT. Published 2017. http://www.quic-concept.eu/

140. Zerka F, Urovi V, Vaidyanathan A, et al. Blockchain for privacy preserving and trustworthy distributed machine learning in multicentric medical imaging (C-DistriM). IEEE Access. 2020;8:183939-183951.

141. Jochems A, Deist TM, El Naqa I, et al. Developing and validating a survival prediction model for NSCLC patients through distributed learning across 3 countries. Int J Radiat Oncol Biol Phys. 2017;99(2):344-352.

142. Shi Z, Zhovannik I, Traverso A, et al. Distributed radiomics as a signature validation study using the Personal Health Train infrastructure. Sci Data. 2019;6(1):218.

143. Bogowicz M, Jochems A, Deist TM, et al. Privacy-preserving distributed learning of radiomics to predict overall survival and HPV status in head and neck cancer. Sci Rep. 2020;10(1):1-10.

144. Shi Z, Traverso A, Soest J, Dekker A, Wee L. Technical note: ontology-guided radiomics analysis workflow (O-RAW). Med Phys. 2019;46(12):5677-5684.

145. https://bioportal.bioontology.org/ontologies/RO

146. Pfaehler E, Zwanenburg A, Jong JR, Boellaard R. RACAT: An open source and easy to use radiomics calculator tool. PLoS One. 2019;14(2):1-26.

147. Nioche C, Orlhac F, Boughdad S, et al. LIFEx: a freeware for radiomic feature calculation in multimodality imaging to accelerate advances in the characterization of tumor heterogeneity. Cancer Res. 2018;78(16):4786 LP-4784789.

148. Fedorov A, Beichel R, Kalpathy-Cramer J, et al. 3D Slicer as an image computing platform for the Quantitative Imaging Network. Magn Reson Imaging. 2012;30(9):1323-1341.

149. Casale R, Lavrova E, Sanduleanu S, Woodruff HC, Lambin P. Development and external validation of a non-invasive molecular status predictor of chromosome 1p/19q co-deletion based on MRI radiomics analysis of low grade glioma patients. Eur J Radiol. 2021;139:109678.

150. Mu W, Katsoulakis E, Whelan CJ, Gage KL, Schabath MB, Gillies RJ. Radiomics predicts risk of cachexia in advanced NSCLC patients treated with immune checkpoint inhibitors. Br J Cancer. 2021. https://doi.org/10.1038/s41416021-01375-0

151. Chiappa V, Interlenghi M, Salvatore C, et al. Using rADioMIcs and machine learning with ultrasonography for the differential diagnosis of myometRiAL tumors (the ADMIRAL pilot study). Radiomics and differential diagnosis of myometrial tumors. Gynecol Oncol. 2021;161:838-844.

152. Zhang F, Zhong L-Z, Zhao X, et al. A deep-learning-based prognostic nomogram integrating microscopic digital pathology and macroscopic magnetic resonance images in nasopharyngeal carcinoma: a multi-cohort study. Ther Adv Med Oncol. 2020;12:1758835920971416.

153. Alvarez-Jimenez C, Sandino AA, Prasanna P, Gupta A, Viswanath SE, Romero E. Identifying cross-scale associations between radiomic and pathomic signatures of non-small cell lung cancer subtypes: preliminary Results. Cancers. 2020;12(12):3663. https://doi.org/10.3390/cancers12123663

154. Geady C, Keller H, Siddiqui I, Bilkey J, Dhani NC, Jaffray DA. Bridging the gap between micro- and macro-scales in medical imaging with textural analysis - A biological basis for CT radiomics classifiers? Phys medica PM an Int J devoted to Appl Phys to Med Biol Off J Ital Assoc Biomed Phys. 2020;72:142-151.

155. FDA. Proposed regulatory framework for modifications to artificial intelligence/machine learning ( $\mathrm{Al} / \mathrm{ML})$-based software as a medical device (SaMD) - discussion paper and request for feedback. US Food Drug Adm. 2019:1-20.

How to cite this article: Guiot J, Vaidyanathan A, Deprez L, et al. A review in radiomics: making personalized medicine a reality via routine imaging. Med Res Rev. 2021;1-15.

https://doi.org/10.1002/med.21846 